\title{
Innovationsfonds - Von der guten Idee zur richtigen Umsetzung
}

VOLKER AMELUNG, SASCHA WOLF

Prof. Dr. Volker Amelung ist Vorstandsvorsitzender des Bundesverbandes Managed Care e.V. in Berlin und Hochschullehrer am Institut für Epidemiologie, Sozialmedizin und Gesundheitssystemforschung an der Medizinischen Hochschule Hannover. Er ist Herausgeber der Zeitschrift „Gesundheits- und Sozialpolitik“

Dr. Sascha Wolf ist Geschäftsführer des Bundesverbandes Managed Care e.V. in Berlin

Die Einrichtung eines Innovationsfonds ist ein
richtiger Schritt zur Weiterentwicklung der

medizinischen Versorgungsstrukturen. Denn wenn wir keine zusätzlichen Impulse für Innovationen im Gesundheitswesen bräuchten, dann müssten wir uns nicht seit fast 40 Jahren über die gleichen Defizite beklagen. Doch so notwendig der Innovationsfonds auch sein mag, so ist seine Einführung gleichermaßen mit Risiken verbunden. Die Erfahrungen mit der Anschubfinanzierung zur Integrierten Versorgung von 2004 bis 2008 zeigen, dass bei falscher Ausgestaltung Fehlanreize gesetzt und Mitnahmeeffekte erzeugt werden. Umso wichtiger ist es, den Regelungsrahmen des Innovationsfonds sorgfältig zu bedenken, einen klaren Kriterienkatalog zu definieren und die Mittelvergabe gleichsam praktikabel wie gezielt zu gestalten.

\section{Einleitung}

Die neue Bundesregierung setzt mit dem Innovationsfonds richtige und notwendige Impulse für mehr Innovation im Gesundheitswesen. Denn die Erfahrung lehrt, dass zusätzliche Anreize benötigt werden, um die erforderliche Weiterentwicklung der Versorgungsstrukturen in der Gesetzlichen Krankenversicherung voranzutreiben. Kritiker wenden aber zu Recht ein, dass hierdurch die Gefahr von Mitnahme- und Gießkanneneffekte entstehen kann. Deshalb ist es umso wichtiger, Konstruktionsfehler wie bei der Anschubfinanzierung zur Integrierten Versorgung von 2004 bis $2008 \mathrm{zu}$ vermeiden und einen Mechanismus zu entwickeln, der einerseits ein Experimentierfeld eröffnet und andererseits die beschränkten finanziellen Fördermittel zielgenau einsetzt. Mit der Verpflichtung zur Evaluation und der Aufstellung eines klaren Kriterienkatalogs tragen die Koalitionäre dieser Gefahr bereits grundsätzlich Rechnung. Jetzt kommt es darauf an, notwendige Konkretisierungen zur praktischen Umsetzung vorzunehmen und unterschiedliche Ausgestaltungsmöglichkeiten sorgfältig zu bedenken. Der vorliegende Beitrag stellt hierzu eine erste Ideensammlung dar.

\section{Warum braucht es mehr Innovation im Gesundheitswesen?}

Das Gesundheitswesen sieht sich einem zweifachen exogenen Kostendruck ausgesetzt (ausführlich hierzu Knieps, Amelung, Wolf 2012). Einerseits wirken sozio-demographische Faktoren auf eine wachsende Nachfrage hin. Andererseits führt die Explosion des Machbaren in der Medizin dazu, dass immer mehr Indikationen behandelbar, aber nicht unbedingt heilbar sind. Bereits heute 
leiden in Deutschland mehr als zehn Millionen Menschen unter chronischen Beschwerden. Das sind 46 Prozent aller Erkrankungen. Viele von ihnen sind multimorbid. Beispielsweise leiden 40 bis 75 Prozent aller depressiven Patienten unter Schmerzsymptomen (Lepine, Briley 2004). Sowohl die Bevölkerungsalterung als auch der medizinisch-technologische Fortschritt werden dazu führen, dass der Anteil der Chroniker bis 2020 um rund 15 Prozentpunkte zunehmen wird (Gensichen et al. 2006). Komplexe Krankheitsbilder stellen besondere Anforderungen an die medizinische Versorgung über die gesamte Wertschöpfungskette hinweg, d.h. vom ambulanten, über den stationären, bis hin in den rehabilitativen und pflegerischen Bereich. Die strikte Sektorentrennung im deutschen Gesundheitswesen ist nicht geeignet, diese Herausforderung effizient zu meistern. Daher bedarf es neuer Versorgungsformen, die eine sektorenübergreifende Koordinierung ermöglichen, Kooperationen zwischen den Leistungserbringern befördern und auch die individuellen Bedürfnisse der Patienten berücksichtigen.

Der demographische Wandel führt nicht nur auf der Nachfrageseite, sondern auch auf der Leistungsseite zu deutlichen Veränderungen. Die Ärzteschaft ist vor der Bevölkerungsalterung ebenso wenig

\section{Ohne zusätzliche Anreize werden die Akteure die erforderlichen Anfangsinvestitionen zur Verbesserung der Versorgung nicht aufbringen.}

gefeit wie die Patienten. Bereits heute ist jeder fünfte Vertragsarzt 60 Jahre und älter (KBV 2012). Bei gleichbleibenden Versorgungsstrukturen schätzt die Kassenärztliche Bundesvereinigung bis 2020 einen „Ersatzbedarf“ von über 70.000 Ärzten. Der drohende Fachkräftemangel wird insbesondere in ländlichen Regionen durch einen spürbaren Mentalitätswandel unter jungen Medizinern verschärft. Nicht nur für Frauen, die mittlerweile mehr als zwei Drittel der Medizinstudenten ausmachen, sondern auch für ihre jungen männlichen Kollegen ist der niedergelassene Landarzt mit 24-Rund-um-Notruf-Service und Wochenendeinsatz schon lange kein
Traumjob mehr (Knieps, Amelung, Wolf 2012). Stattdessen werden kooperative Arbeitsstrukturen und Angestelltenverhältnisse zunehmend präferiert.

Vor diesem Hintergrund müssen die traditionellen Strukturen auf den Prüfstand gestellt und weiterentwickelt werden. Neue kooperative Organisationsmodelle wie Ärztenetze und Medizinische Versorgungszentren, indikations- und populationsorientierte Versorgungsstrukturen, arztentlastende Delegation und Substitution sowie neue Mobilitätskonzepte und Technologien können entscheidend dazu beitragen, Behandlungsprozesse zu optimieren, dem drohenden Fachkräftemangel entgegenzuwirken und die flächendeckende Versorgung vor Ort auch zukünftig zu gewährleisten.

\section{Warum braucht es Innovationsförderung?}

Die strukturellen Defizite der medizinischen Versorgung in der Gesetzlichen Krankenversicherung sind seit 40 Jahren bestens bekannt (vgl. Jahn 1975). Die Erfahrung lehrt, dass die Akteure des Gesundheitswesens ohne zusätzliche Anreize die erforderlichen Anfangsinvestitionen zur Verbesserung der Versorgungsorganisation nicht aufbringen werden. Dabei ist es nicht die einzelne Krankenkasse, der einzelne Arzt oder der einzelne Patient, der sich aus intrinsischen Motiven Veränderungen verweigert. Es sind die institutionellen Rahmenbedingungen, die entscheidend sind für die Lethargie eines solch komplexen Systems - in den Wirtschaftswissenschaften spricht man von Pfadabhängigkeiten (vgl. Schreyögg, Sydow, Eberl 2008) - wie das der Gesetzlichen Krankenversicherung (ausführlich hierzu Amelung, Wolf 2013). Folgen sind, dass die Verhandlungs- und Versorgungsstrukturen vermachtet und starr sind und der Anteil der Ausgaben für Integrierte Versorgung an den GKVGesamtausgaben bei unter einem Prozent stagniert (SVR 2012, S. 349).

Der Gesetzgeber hat in den vergangenen Jahren durchaus reagiert und mit zahlreichen Handlungsspielräumen und Vertragsfreiheiten den Nährboden für mehr Innovationen im System bereitet. Die Akteure haben ihrerseits ihre Kreativität unter Beweis gestellt und eine große
Spannbreite innovativer Pilotprojekte auf den Weg gebracht. Doch spätestens dann, wenn es darum geht, Modelle, die nachweislich die Kosten senken oder die Qualität steigern, zu multiplizieren und in die Fläche zu tragen, stoßen die Initiatoren auf oftmals unüberwindliche Hürden. Der Nährboden allein ist demnach nicht ausreichend. Die zentrale Schwäche des Gesundheitswesens ist das bislang fehlende, doch zum Gedeihen notwendige investitionsfreundliche Klima.

Zur Herstellung eines solchen Klimas kann der Innovationsfonds einen wertvollen Beitrag leisten. Vergleichbar mit der Förderung von Start-up-Unternehmen beispielsweise in der IT- und Hightechgründerszene können hierdurch die unternehmerische Risiko- und Investitionsbereitschaft potenzieller Financiers erhöht, erforderliche Anschubfinanzierungen von den Projektinitiatoren leichter generiert und somit neue Chancen für innovative Geschäftsmodelle und Versorgungsstrukturen eröffnet werden. Die Erhöhung der Investitionsfähigkeit ist jedoch nur eine Seite der Medaille. Auf der anderen Seite müssen noch immer bestehende rechtliche Hürden für neue Versorgungsformen beseitigt und ein niedrigschwelliger Zugang für Innovationen ins System geschaffen werden. Deshalb ist es richtig, dass die Bundesregierung die Rahmenbedingungen für die unterschiedlichen integrierten und selektiven Versorgungsformen angleichen und Investitionshemmnisse abbauen möchte.

\section{Koalitionsvertrag legt Stoßrichtung fest - Konkretisierungen müssen folgen}

Im Koalitionsvertrag kündigt die Bundesregierung die Einrichtung eines Innovationsfonds in Höhe von 300 Millionen Euro an, der von den Krankenkassen zur Verfügung gestellt werden soll. Die Hälfte des Finanzvolumens refinanziert sich über zusätzliche Zuweisungen aus den Liquiditätsreserven des Gesundheitsfonds. 225 Millionen Euro sind für Versorgungsleistungen, die über die Regelversorgung hinausgehen, vorgesehen. Die übrigen 75 Millionen Euro sollen der Versorgungsforschung zugutekommen. Die Vergabe der Mittel erfolgt im Rahmen eines jährlichen Ausschreibungsverfahrens durch den Gemeinsamen Bundesausschuss (G-BA), der hierzu vorab Kriterien definiert. Eine Evaluierung der 
geförderten Projekte ist nach vier Jahren vorgesehen (Koalitionsvertrag 2013).

Mit diesen Ankündigungen legt die Bundesregierung bereits eine erstaunliche Detailtiefe an den Tag. Dennoch bleiben zahlreiche Fragen zur konkreten Umsetzung unbeantwortet. Im Folgenden sollen daher mögliche Gestaltungsoptionen diskutiert werden.

\section{a) In welchem Umfang und wie lange soll gefördert werden?}

Unter der Annahme, dass es sich bei den genannten 300 Millionen Euro um einen jährlichen Betrag handelt, ist das Volumen großzügig bemessen - zumal es allein um die Förderung zusätzlicher Leistungen geht. Somit handelt es sich um eine im internationalen Vergleich einzigartige Unterstützung neuer Versorgungsformen. Zwar bewegt sich die Bundesregierung damit unterhalb der Höhe der Anschubfinanzierung von 2004 bis 2008. Die gezieltere Vergabe der Gelder

\section{Damit ein breites} Experimentierfeld geschaffen und Wettbewerbsverzerrungen vermieden werden, sollten Förderhöchstgrenzen definiert werden.

stellt jedoch sicher, dass ausreichend freie Mittel zur Verfügung stehen. Der Vorwurf einer zu geringen Ausstattung des Innovationsfonds kann somit sicherlich nicht erhoben werden.

Entscheidender als die absolute Höhe des Fördervolumens ist die Verteilung der Gelder. Damit ein breites Experimentierfeld geschaffen werden kann und Wettbewerbsverzerrungen durch massive Zuweisungen an einige wenige Projekte vermieden werden, sollten Förderhöchstgrenzen definiert und nur Teilfinanzierungen (z.B. eine Förderquote von 50 Prozent) vorgenommen werden, wie es auch beim Forschungsrahmenprogramm der EU üblich ist. Zudem sollten die Zuweisungen von vornherein zeitlich begrenzt bleiben. Wichtig ist, dass die Projektinitiatoren immer auch einen Teil des unternehmerischen Risikos tragen. Dies wirkt Mitnahmeeffekten entgegen und gewährleistet, dass nur Förderanträge zu Modellen gestellt werden, die eine realistische Aussicht auf einen zumindest mittelfristig positiven Return-on-Investment haben.

\section{b) Welche Versorgungsleistungen sollen gefördert werden?}

Der Grundgedanke, ausschließlich Versorgungsleistungen zu fördern, die über

die Regelversorgung hinausgehen, ist an der Regelversorgung sind, desto mehr stellt sich die Problematik der Budgetbereinigung, für die - mit Ausnahme der Hausarztzentrierten Versorgung komplexer Bereinigungsberechnungen umgangen werden.

Der Koalitionsvertrag bleibt bislang jedoch eine Antwort auf die Frage schulüber den Innovationsfonds zum bereits vorhandenen, im SGB V geregelten Inonsleistungen einbezogen werden. Hierzu Managementleistungen und die notwendige IT-Infrastruktur. Nicht zuletzt spielt auch die Aneignung der jeweils erforderliDecision-Making basiert, dann muss auch zielführend. Denn je dichter die Projekte bis heute keine praktikable Regelung existiert. Durch die Fokussierung auf „Add-on“-Leistungen können Doppelfinanzierungen ohne Implementierung dig, in welchem Verhältnis die Förderung

\section{c) Was heißt Förderung der Versorgungsforschung?}

Die Versorgungsforschung bildet das Fundament jeglichen Versorgungsmanagements. Die Zweiteilung des Innovationsfonds geht daher in die richtige Richtung. Ziel sollte jedoch weder ein akademisches Sonderforschungsprogramm universitärer Einrichtungen noch eine Alternative oder gar ein Ersatz bereits vorhandener Förderprogramme beispielsweise des Bundesministeriums für Bildung und Forschung sein. Stattdessen muss es darum gehen, die basisnahe Versorgungsforschung innerhalb der an der praktischen Umsetzung der Projekte beteiligten Institutionen zu unterstützen. Aus Sicht der Krankenkassen und deren Partner ist Versorgungsforschung immer auch eine partielle Marktforschung. Es geht darum, Erkenntnisse darüber zu erhalten, wie Versorgung in der Praxis funktioniert, um darauf aufbauend effiziente Versorgungsprozesse gestalten zu können. Mögliche Themenfelder sind u.a. der Aufbau einer zugänglichen Datenbank für Routinedaten oder die Entwicklung standardisierter Evaluationskonzepte.

\section{d) Wer soll gefördert werden?}

Laut Koalitionsvertrag wird das Geld von den Krankenkassen zur Verfügung gestellt. Damit bleibt die Frage jedoch unbeantwortet, wer zum Kreis förderungswürdiger Organisationen zählt. Um das größtmögliche Innovationspotenzial auszuschöpfen bietet es sich an, allen Akteuren des Gesundheitswesens ein Antragsrecht zuzugestehen. Wer sich in der Lage sieht, Versorgungsprozesse $\mathrm{zu}$ entwickeln oder zu verbessern, z.B. Leistungserbringer, Konsortien von Leistungserbringern oder Managementgesellschaften, sollte dies auch tun können.

\section{e) Wer entscheidet über die Mittelvergabe?} gehören die Gestaltung der Prozesse, die chen Kernkompetenzen eine entscheidende Rolle. Wenn z.B. ein Vertrag auf Shared-
Die Mittelvergabe und Durchführung des Ausschreibungsverfahrens soll durch den G-BA erfolgen. Aus politischer Sicht besitzt diese Vorgehensweise durchaus eine gewisse Attraktivität: Zum einen wird hierdurch die Aufstellung klarer, für alle Beteiligten relevanter Kriterien 
und ein hoher Grad an Transparenz ermöglicht. Zum anderen besitzt der G-BA durch seine Grundstruktur, in der neben den Krankenkassen und den Leistungserbringern zumindest in beratender Funktion auch die Patienten vertreten sind, eine breite Legitimationsbasis. Nicht zuletzt kann auf eine vorhandene, professionelle Infrastruktur zurückgegriffen werden. Aus Wettbewerbssicht muss die Kompetenzstärkung des G-BA jedoch kritisch hinterfragt werden. So besteht die Gefahr von interessengeleiteten Vergabeentscheidungen. Im G-BA sind Personen bzw. Institutionen vertreten, die unmittelbar oder mittelbar von den Projekten selbst betroffen sind. Die Definition von verlässlichen, einsehbaren Regeln für die Mittelvergabe kann dieses Risiko abschwächen, aber nicht beseitigen. Besser wäre es daher, die Vergabekompetenz an ein neutrales Gremium, beispielsweise einer Stiftung, zu übertragen. Das Kuratorium könnte sich aus Vertretern verschiedener Ministerien und gesellschaftlich anerkannter Personen, die selbst nicht Akteure des Versorgungsgeschehens sind, zusammensetzen. Dabei darf jedoch nicht aus dem Blick geraten, dass es sich generell um Beitragsgelder handeln wird und entsprechend mit ihnen umgegangen werden muss.

\section{f) Wie funktioniert das Ausschreibungsverfahren?}

Bereits existierende Ausschreibungsverfahren im Gesundheitswesen zeichnen sich oftmals durch einen hohen Grad an Komplexität aus. Umfangreiche Exposés sind mit entsprechend hohen Kosten für die Antragsteller verbunden. Um zu verhindern, dass weniger finanzstarke Projektinitiatoren von vornherein an den gestellten Anforderungen scheitern, könnte ein zweistufiges Verfahren in Erwägung gezogen werden. Auf der ersten Stufe sollte ein Kurzdossier, beispielsweise von maximal 10 Seiten, ausreichen. Weitere Details könnten auf der zweiten Stufe des Verfahrens angefordert werden. Eine Standardisierung der einzureichenden Unterlagen würde zur Vereinfachung und Transparenz beitragen.

Um eine zeitnahe Förderung zu gewährleisten sollte an Stelle einer jährlichen Ausschreibung ein halbjähriges Verfahren durchgeführt werden. Dies würde die Zeitspanne zwischen Pro- jektidee und Umsetzung verkürzen und damit zur Planungssicherheit beitragen. Zudem sollte die Ablehnung eines Projekts nicht zum endgültigen Ausschluss führen. Die Möglichkeit zur Wiederbewerbung würde die Chance für Nachbesserungen eröffnen.

\section{g) Wie soll evaluiert werden?}

Eine Verpflichtung zur Evaluation ist zur Verhinderung von Mitnahmeeffekten unabdingbar. Der im Koalitionsvertrag angegebene Zeitrahmen von vier Jahren ist aus doppelter Sicht sinnvoll: Zum einen ist es vorher kaum möglich, belastbare Evaluationsergebnisse zu erzielen. Zum anderen werden hierdurch insbesondere die Krankenkassen von kurzfristigen Betrachtungen befreit. Durch die Kombination aus Teilfinanzierung über den Gesundheitsfonds und die Vier-Jahres-Frist erhalten die Kassen Planungssicherheit und die Möglichkeit zur Umsetzung langfristiger Projekte.

Um eine hohe Qualität der Evaluationen sicherzustellen, sollte das entsprechende Assessmentverfahren bereits bei der Mittelvergabe feststehen. Denn Evaluationen besitzen nur dann Aussagekraft, wenn sie zeitgleich mit dem zu bewertenden Projekt gestartet werden. Um darüber hinaus die Vergleichbarkeit der Evaluationen unterschiedlicher Projekte zu gewährleisten, muss im Vorhinein ein einheitlicher Kriterienkatalog entwickelt werden. Eine solche Standardisierung könnte über eine Festlegung der Methoden, der Mindeststandards und Signifikanzlevels erfolgen.

\section{Fazit}

Eine gute Idee ist die Voraussetzung, aber noch kein Garant für ein funktionierendes Konzept. Richtig ausgestaltet kann der von der Bundesregierung angekündigte Innovationsfonds einen spürbaren Beitrag zur Verbesserung des Investitionsklimas im deutschen Gesundheitswesen leisten. Aus diesem Grund empfehlen Institutionen wie der Bundesverband Managed Care e.V. seit vielen Jahren die Förderung neuer Versorgungsformen. Dabei darf die Gefahr von Fehlanreizen jedoch nicht übersehen werden. Der konkreten Umsetzung kommt deshalb entscheidende Bedeutung zu. Die Politik hat mit dem Innovationsfonds einen ebenso wichtigen wie richtigen Schritt eingeleitet. Doch sie kann nur den Rahmen zur Verfügung stellen. Jetzt sind die Akteure des Gesundheitswesens an der Reihe. Wenn Selbstverwaltungsstrukturen legitimiert sein wollen, dann müssen die Akteure ihre Investitionsbereitschaft und Innovationsfähigkeit unter Beweis stellen. Es liegt an Ihnen, den Impuls der Politik aufzunehmen und in einen dauerhaften Antrieb umzuwandeln.

Letztlich stellt der Innovationsfonds immer nur eine Second-Best-Lösung dar. Messlatte ist aber nicht eine von Ökonomen gerne angepriesene Idealwelt, sondern die aktuelle Versorgungsrealität. Hier setzt der Fonds deutliche Anreize für mehr Effizienz und Qualität. Doch Anreize allein verändern nichts, sondern nur die durch sie bewirkten Taten. Es liegt nun an uns allen, Kreativität, Mut und Unternehmergeist an den Tag zu legen.

\section{Literatur}

Amelung V / Wolf S: Innovationsförderung in der GKV. Gesundheits- und Sozialpolitik, 2-3/2013, S. $111-117$.

Gensichen J / Muth C / Butzlaff M / Rosemann T / Raste H / Müller de Cornejo G et al. (2006): Die Zukunft ist chronisch - Das Chronic Care Modell in der deutschen Primärversorgung. Zeitschrift für ärztliche Fortbildung und Qualität im Gesundheitswesen, 10

Jahn E (1975): Systemanalyse der medizinischen Versorgung in der Bundesrepublik. In: Integrierte medizinische Versorgung: Notwendigkeiten - Möglichkeiten - Grenzen, WSI-Forum am 25. und 26. April 1975. WSI-Studie Nr. 32, Bund-Verlag Köln.

KBV (Kassenärztliche Bundesvereinigung) (2012): Grunddaten 2011 zur vertragsärztlichen Versorgung in Deutschland, Berlin.

Knieps F / Amelung V / Wolf S (2012): Die Gesundheitsversorgung in schwer zu versorgenden Regionen - Grundlagen, Definition, Problemanalyse. Gesundheits- und Sozialpolitik, 6/2012, S. 8-19.

Koalitionsvertrag (2013): Deutschlands Zukunft gestalten. Koalitionsvertrag zwischen CDU, CSU und SPD, 18. Legislaturperiode, Berlin.

Lepine JP / Briley M (2004): The epidemiology of pain in depression. Hum Psychopharmacol, 19/2004, Suppl. 1, S. $3-7$.

Schreyögg G / Sydow J / Eberl M (2008): Vom Innovationsvorsprung zur Kompetenzfalle: Strategische Pfade in Unternehmen, Innovationsforum der Gottlieb Daimler- und Karl Benz-Stiftung, 26. Mai 2008.

SVR (Sachverständigenrat zur Begutachtung der Entwicklung im Gesundheitswesen) (2012): Sondergutachten 2012 - Wettbewerb an der Schnittstelle zwischen ambulanter und stationärer Gesundheitsversorgung, Verlag Hans Huber Hogrefe. 